Sharif University of Technology
Scientia Iranica
Transactions E: Industrial Engineering
hCIENTIA

\title{
Multi-objective mathematical modeling of an integrated train makeup and routing problem in an Iranian railway company
}

\author{
R. Alikhani-Kooshkak ${ }^{a}$, R. Tavakkoli-Moghaddam ${ }^{\mathrm{b}, c^{*}}$, A. Jamili ${ }^{\mathrm{b}}$, and \\ S. Ebrahimnejad ${ }^{\text {d }}$ \\ a. School of Industrial Engineering, South Tehran Branch, Islamic Azad University, Tehran, Iran. \\ b. School of Industrial Engineering, College of Engineering, University of Tehran, Tehran, Postal Code: 1439957131, Iran. \\ c. Arts et Métiers ParisTech, LCFC, Metz, France. \\ d. Department of Industrial Engineering, Karaj Branch, Islamic Azad University, Karaj, Iran.
}

Received 10 June 2017; received in revised form 10 March 2018; accepted 21 July 2018

\section{KEYWORDS}

Train makeup and routing problem;

Optimization with multiple objectives; $L_{p}$ norm;

Goal-oriented optimization (GP); Simulated annealing.

\begin{abstract}
Train formation planning faces two types of challenges, namely the determination of the number of cargo trains run known as the frequency of cargo trains and the formation of desired allocations of demands to a freight train. To investigate the issues of train makeup and train routing simultaneously, this multi-objective model optimizes the total profit, satisfaction level of customers, yard activities in terms of the total size of a shunting operation, and underutilized train capacity. In addition, this model considers guarantee for the yard-demand balance of flow, maximum and minimum limitations for the length of trains, maximum yard limitation for train formation, maximum yard limitation for operations related to shunting, maximum limitation for the train capacity, and upper limit of the capacity of each arc in passing trains. In this paper, a goal programming approach and an $L_{p}$ norm method are applied to the problem. Furthermore, a Simulated Annealing (SA) algorithm is designed. Some test problems are also carried out via simulation and solved using the SA algorithm. Furthermore, a sample investigation is carried out in a railway company in Iran. The findings show the capability and performance of the proposed approach to solve the problems associated with a real rail network.
\end{abstract}

(C) 2019 Sharif University of Technology. All rights reserved.

\section{Introduction}

Dependent on policy, sustainability, and environment, transportation is believed to have a significant role in improving economic conditions of a country leading to enhanced economic indices, such as Gross Domestic

\footnotetext{
*. Corresponding author. Tel.: +982182084183

E-mail addresses: tavakoli@ut.ac.ir and

reza.tavakkolimoghaddam@ensam.eu ( $R$.

Tavakkoli-Moghaddam).
}

doi: $10.24200 /$ sci. 2018.20782
Product (GDP) [1,2]. Recently, new development opportunities for various industries and service sectors have been made possible by railway transportation.

Railway enjoys significant advantages over other methods of transportation because it is able to respond to freight demand at larger distances in terms of length as demanded with a higher safety degree [3]. Empirical findings based on indicators and performances show the concern for the operational optimization of a railway network based on important objectives such as economy, efficiency, and customer satisfaction. Consequently, among the various studies on railway transportation, a growing body of evidence 
from the transportation literature shows that a Train Makeup Problem (TMP) is of remarkable importance to academia and practitioners. Hence, the TMP has gained significance in railway transportation planning investigations. Among the various goals of optimization, an important objective has received the main attention, in which the necessary trains are formed to satisfy demand in a desired period of time. In addition, such an optimization model considers the operational and physical constraints of the network to achieve feasible and optimal sizes, frequencies, assignments, traction, and demand profiles regarding the specific objective functions [4]. However, due to NP-hardness issues, the main challenge remains: dealing with the difficulty of solving the train makeup problem in the context of a design problem of a service network.

Some new features are added to the railway planning literature in this research. In some railway transportation networks, there may be different routes from each origin to a destination. A generic routing problem tries to determine an optimal route to provide an efficient routing plan. A routing problem in the TMP is not taken into account in literature reviews; therefore, a novel optimization model with multiple objectives is considered based on integrating the makeup train and routing problems all together. In comparison to the current literature, some new constraints are added to the optimization model, which improve its validity in order to provide a better basis of reflecting real-world conditions. Following the common stream of research in multi-objective optimization, Goal Programming (GP) and $L_{p}$ norm methods are applied. In addition, a new simulated annealing algorithm is proposed to provide good solutions for the problem. Considering the application of the algorithm, ten randomly designed problems, as well as a real-world problem, in the context of a railway company in Iran are tested by the algorithm. To compare them by the CPLEX standard software, some of the results from the algorithm, which are more pertinent, are checked for greater efficiency in comparison with those obtained from the software.

Several sections contribute to the organization of the paper. Studies related to the TMP are reviewed in Section 2. Section 3 presents a model for the train makeup and routing problem based on a new approach considering new multiple objective functions. Following the main optimization research stream, Section 4 applies two important optimization methods to find a single objective function to replace with the multiple objective functions of the model. Section 5 reports the results of applying the solution method to some randomly generated test problems and provides optimality and sensitivity analysis findings. Based on a hybrid meta-heuristic, Section 6 proposes a new algorithm by developing a novel hybrid sim- ulated annealing algorithm. Section 7 presents the computational findings of applying the algorithm to 10 randomly generated test problems and a real-world problem associated with a railway company in Iran. As the final section, Section 8 concludes the paper and provides some further research remarks.

\section{Literature review}

A TMP has received the attention of researchers from a mathematical point of view. For example, Assad [5] presented a multi-commodity network flow model to analyze a TMP. To investigate tactical planning, in a programming model with a mixed-integer and nonlinear nature solved by a heuristic method, Crainic et al. [6] minimized delay and operating costs. Based on a heuristic approach and a Lagrangian relaxation technique, Keaton [7] developed a method to deal with a programming model characterized by a mixed-integer structure. Morlok and Thomas [8] developed a programming model with a mixed-integer linear structure and used a branch-and-bound technique to solve some small-sized instances. Huntley et al. [9] presented an MINLP model and used a SA algorithm to find a near-optimal solution. Adopting a hybrid approach, Yaghini et al. [4] designed a SA based on simplex for the TMP, in which the moves in this method are evaluated, selected, and implemented. Bagheri et al. [10] proposed a method for placing hazardous material cars to minimize the derailment risk during the train assembly process. With respect to yard activities, they took into account the chance associated with derailing of railway cars with the route and the probability of extra activities.

Shafia et al. [11] developed a novel robust model with a mixed-integer nature for a TMP with uncertain input data, in which a heuristic method is designed because of the difficulty of finding an optimal solution. Based on the train scheduling and with emphasis on the average travel duration time, Sun et al. [12] considered the user's satisfaction and energy consumption to present an optimization model with multiple objective functions for the train routing on a railway network supporting high speed, which is able to provide an important basis for a train planning to provide a good service. For implementation purposes, an enhanced Genetic Algorithm (GA) was developed and implemented. Yaghini et al. [3] developed a hybrid technique to solve a mathematical model for train makeup planning with fuzzy costs in an Iranian railway system. In fact, they developed a network design model that considered a fixed-charge situation with capacity limitations and multiple commodities. In an operational setting on a railway network, Masek et al. [13] mathematically investigated the transportation of single-wagon consignments. Borndörfer et al. [14] 
analyzed a problem that considers the freight train routing. Considering both passenger and cargo, they investigated a transport network, in which routes of trains are fixed. Taking into account all running times and all expected delays for each freight train, they found feasible and optimal routes. Boysen et al. [15] introduced and discussed the train formation problem in its basic setting. They analyzed the status of its complexity and designed both heuristic and exact solution methods. They also tested their solution method in a wide-ranging computational experiment. Cheng et al. [16] proposed a methodology to assess risk. Adopting a mathematical approach, they modeled the train formation and the location of railcars of hazmat kind in a predefined transport passage. A sample study was considered and analyzed to gain managerial insights with four different sets of conditions related to an American network.

Based on programming with a mixed-integer nature, Bahrami et al. [17] integrated hub location and vehicle routing problems in order to provide door-todoor parcel delivery services. A simulated annealing approach helped them propose a novel technique that has multiple steps based on an algorithm with local search capability. They used the technique to solve a real case in Iran. Gallardo-Bobadilla and Doucette [18] applied a linear programming approach to optimize the railway blocking problem efficiently and find the optimal allocation of shipments to blocks and their routing plan with an acceptable level of error. Yaghini et al. [19] proposed a modified specific method for a train makeup problem. This is an exact approach in real life of the railway network. This method is proposed to modify the behavior of a standard solver to deal with a mixed-integer programming problem.

Jamili [20] focused on trip duration and expected deferrals and presented a novel periodic train-scheduling mathematical model under uncertainty. He proposed Simulated Annealing (SA) and Electromagnetism-like Method (EM), as well as a hybrid of these two metaheuristics, for solving the problem. To deal with an integer-linear setting, TavakkoliMoghaddam et al. [21] designed a programming model for a cell formation problem in an environment that is dynamic with a multi-cycle scheming vision. This problem is categorized as an NP-hard problem; thus, they took into account an SA algorithm to solve their model. Furthermore, Alikhani-Kooshkak et al. [22] presented a multi-objective model for the train makeup problem with locomotive limitations. They considered seven different objectives (e.g., minimizing the total lost demand, the transfer time of trains, and the total consumed fuel) and proposed a hybrid firefly algorithm to solve the problem in a real-word application.

Despite its multi-dimensional nature, the litera- ture review reveals that the multi-objetive optimization of the TMP is understudied. Some examples are offered here. First, maximization of the profit is possible via mathematical focus on incomes and expenses. Second, the maximization of customers' satisfaction level is attainable by considering frequencies of trains. This results in the decrease of the time demands spent in yards for shunting activities. Thus, one criterion for the maximization of customers' satisfaction level and minimization of the costs is to assume maximizing trains in terms of the total frequencies, minimizing yard activities in terms of the total quantity of the actions of shunting kind, and minimizing train underutilization.

This is currently missing from the literature (see e.g. $[3,4])$; basically, the minimization of variable and fixed costs is common in the current literature. In addition, all the required constraints associated with train formation problem are not considered in the current stream of research. Focusing on the length of the train, Yaghini et al. [19] modeled merely the biggest train considering the size of station with respect to the number of trains and the quantity of actions of shunting kind. In other words, they did not consider significant concepts such as the determined minimum limitation related to the least length of each train which is formed, train tonnage, the capacity of yard in terms of tonnage, available time, and upper limit of the capacity of each arc for train passage through different routes in the railway network.

\section{Mathematical model}

In this section, the fixed and variable costs of transportation are optimized. In doing so, we assume the transportation of goods with a specific origin and destination for each train based on available information (e.g., [19]). Here, the origin and destination define each train. We also assume that there exists a balance of the flow for each yard-demand pair. Furthermore, we consider that there is an upper limit for the length of train that constrains the amount of demand allowed to be allocated to an arbitrary train. Each yard faces a maximum limitation in the number of trains and another maximum limitation in the size of demands put together for train formation in an operation with a classification or shunting nature. The current literature is extended by adding some new features. First, a routing problem with the train makeup in railway networks is assumed. In some railway transportation networks, there may be different routes from each origin to a destination. Each route of a network consists of some tracks (i.e., arcs), in which each track has a specific capacity (i.e., a number of trains passing through the track in a specific time period). Additionally, one of the 
main contributions of this presented model is the transfer of demands from an origin to a destination via different routes consisting of tracks (i.e., arcs) with the limited capacity. It is worth noting that a decision for a train's route is made based on the objectives of our integrated train makeup and routing problem and related limitations. In fact, this model determines the trains and relevant routes in order to transport dedicated demand. Notably, the proposed model specifies that each demand must be transferred by which trains. It is worth noting that the basic model [19] does not cover this topic. It may not be economical to form a train to carry almost nothing due to a very low volume of demands. In addition, the capacity of routes in terms of tonnage and the arcs capacity in terms of the size of frequencies of passing trains via arcs are constrained. Therefore, this paper considers some new constraints for the original model to extend it in order to improve its validity by considering previously ignored important real-life conditions. In particular, a minimum limitation for the length of train is assumed to constrain the least amount of demands that can be allocated to a train. A maximum limit is considered in terms of tonnage for the capacity of yard operations of shunting type. Moreover, a maximum limitation for the number of trains is assumed that allows for passing through an arbitrary arc of the network.

Second, in the basic model, all transportationbased requests are accepted. In contrast, the lost demand property is allowed in our model, and the costs and benefits of accepting a request are compared. This leads to denial of some demands due to their uneconomical nature. These directions are not considered in the previous literature.

In this paper, four objective functions are considered by optimizing the profit and customers' satisfaction level to its maximum possible, yard activity in terms of the total quantity of the operations of shunting kind, and the underutilized trains in terms of tonnage to its minimum possible.

Based on the above points, a mathematical approach is adopted to formulate a novel model below.

\section{Notations}

$k$

Demand $k$ belongs to the set $K$

Train $t$ belongs to the set $T$

Yard $s$ belongs to the set $S$

Route $r$ belongs to the set $R$

Arc $i$ belongs to the set $I$

$K \quad$ Set $K$ denotes demands originating from an arbitrary node called orig $(k)$ and destined to $\operatorname{dest}(k)$
Set $T$ denotes trains carrying demands. Each train starts from its origin and ends at its destination in a setting where its origin and destination have been set before. The train does not load or unload any demand in the midway; however, it is allowed to take many paths in its entire trip.

Set $S$ denotes yards. Each yard receives trains, breaks them up, blocks their cars, assembles their demand, and forms the train. After these activities, the train is ready to depart from the station.

$R \quad$ Set $R$ denotes the routes between the Origin and Destination (O-D) of trains. Each route contains a number of arcs

$I \quad$ Set $I$ denotes the arcs in the related railway network

$\operatorname{orig}(k) \quad$ Demand $k$ originates from node orig $(k)$

$\operatorname{dest}(k) \quad$ Demand $k$ is destined to node $\operatorname{dest}(k)$

$G_{k} \quad$ One unit of demand $k$ has the weight $G_{k}$

$b_{k} \quad$ If the rail company ships demand $k$, it gain $b_{k}$ for each unit of demand

$d_{k} \quad$ Amount of flow $d_{k}$ from its origin $(\operatorname{orig}(k))$ to its destination $(\operatorname{dest}(k))$

$c_{t}^{k} \quad$ Shipping one unit of demand $k$ on train $t$ has the cost $c_{t}^{k}$

$f_{t}^{r} \quad$ Forming train $t$ on route $r$ has a fixed cost $f_{t}^{r}$

$U_{t}^{r} \quad$ Train $t$ on route $r$ has a maximum $U_{t}^{r}$ of units of demand allocated to it. This concept helps define the biggest train in terms of length

$L_{t}^{r} \quad$ Train $t$ on route $r$ has a minimum $L_{t}^{r}$ of units of demand allocated to it. This concept helps define the smallest train in terms of length

$P_{t}^{r} \quad$ Train $t$ on route $r$ has the capacity $P_{t}^{r}$ in terms of tonnage

$Q_{s} \quad$ Shunting operation in yard $s$ has the capacity $Q_{s}$ in terms of tonnage

$H_{s} \quad$ Trains formed in yard $s$ have the maximum number $H_{s}$

$N_{s} \quad$ Shunting operations performed in yard $s$ have the maximum number $N_{s}$

$A_{i} \quad$ For a desired period of planning, trains passing through arc $i$ have the maximum number $A_{i}$

$c_{k} \quad$ Rail company loses $c_{k}$ for each unit of demand if it rejects demand $k$ 
$a$

Planner is allowed to reject the fraction $a$ of demands as the lost sale ratio

\section{Decision variables}

$x_{t}^{k} \quad$ Demand $k$ on train $t$ has the flow amount of $x_{t}^{k}$

$y_{t}^{r} \quad$ Train $t$ on route $r$ has the number of frequencies $y_{t}^{r}$

$w_{k} \quad$ Demand $k$ has the amount $w_{k}$ as the number of lost units from transporting. In assessing a request, the rail company compares both benefits and costs of accepting a request

\subsection{Mathematical model}

\subsubsection{Objective functions}

In this section, the objective functions of our model are explained. The first objective function optimizes profit. It attempts to maximize income. In addition, it attempts to minimize the costs. Variable, fixed, and lost demand costs are considered in the model. The second objective function optimizes customers' satisfaction level. It attempts to maximize the total train frequencies. If the total train frequencies increase, a decrease in demands waiting in yards of shunting kind prepared to be transferred from an origin to a destination is expected; consequently, one criterion for the improvement of customers' satisfaction level is perhaps maximizing the total train frequencies. The third function targets the minimum of the yard activities in terms of the total number of operations of shunting kind. To put it differently, the main objective here is to minimize the unnecessary disassembly of wagons (i.e., demands) in intermediate stations before arriving at the destination targeted by the demand. In fact, this approach leads to the minimization of trainwise transportation demands with mismatching origins and destinations. The best scenario occurs when two conditions are satisfied: (a) the origin of demand and the origin of the train carrying the demand coincide and $(b)$ the destination of demand and that of the train carrying the demand coincide. This implies that the optimal plan to transport a demand to avoid unnecessary shunting costs and operations requires a single train, which carries the demand throughout its journey from its origin to its destination. The fourth objective function targets the minimum of underutilized trains. It attempts to find minimum differences between the trains in terms of tonnage able to be carried and the demands, which are transferred.

$$
\begin{aligned}
\max Z_{1}= & \sum_{k} b_{k}\left(d_{k}-w_{k}\right)-\sum_{k} \sum_{t} c_{t}^{k} x_{t}^{k} \\
& -\sum_{t} \sum_{r} f_{t}^{r} y_{t}^{r}-\sum_{k} c_{k} w_{k},
\end{aligned}
$$

$$
\begin{aligned}
& \max Z_{2}=\sum_{t} \sum_{r} y_{t}^{r}, \\
& \min Z_{3}=\sum_{s} \sum_{\begin{array}{c}
k \\
\text { dest }(k) \neq \operatorname{dest}(t) \\
\operatorname{dest}(t)=s
\end{array}} \sum_{t} x_{t}^{k}, \\
& \min Z_{4}=\sum_{t} \sum_{r} P_{t}^{r} y_{t}^{r}-\sum_{k} \sum_{t} G_{k} x_{t}^{k} .
\end{aligned}
$$

\subsubsection{Limitations considered in the model}

The lost demand is allowed in transportation. Constraint (5) establishes the balance of flows for all of the yard-demand pairs. Constraint (6) requires the predetermined maximum limit for the trains that are formed in terms of its length to constrain the uppermost quantity of the demands allocated to trains. Inequality (7) requires the predetermined maximum limit for the number of trains formed in all the yards. Constraint (8) requires the predetermined maximum limit for the yard activity in terms of the number of the operations of shunting kind. Constraint (9) examines the predetermined minimum limit for each formed train in terms of its length. Constraint (10) demonstrates the capacity of train in tonnage as the maximum limit. It models the maximum weight the train is allowed to carry. Inequality (11) ensures that each yard shunts demands as much as its shunting capability in terms of tonnage. For a desired period of planning, Inequality (12) necessitates the predetermined maximum limit for the overall frequencies of trains passing each track. Constraint (13) defines the magnitude of demands permitted to be lost since they are rejected and will not be transported. Since variables $x, y$, and $w$ are required to be integer, Constraint (14) imposes this requirement.

$$
\begin{aligned}
& \sum_{\operatorname{orig}(t)=i} x_{t}^{k}-\sum_{\begin{array}{c}
t \\
\operatorname{dest}(t)=i
\end{array}} x_{t}^{k}= \\
& \left.\qquad \begin{array}{ll}
d_{k}-w_{k} & \operatorname{orig}(k)=i \\
-d_{k}+w_{k} & \operatorname{dest}(k)=i \\
0 & \text { otherwise }
\end{array}\right\}
\end{aligned}
$$

$\forall i \in S, \quad k \in K$

$\sum_{k} x_{t}^{k} \leq \sum_{r} U_{t}^{r} y_{t}^{r} \quad \forall t \in T$,

$\sum_{\substack{t \\ \operatorname{Orig}(t)=s}} \sum_{r} y_{t}^{r} \leq H_{s} \quad \forall s \in S$,

$\sum_{\substack{k \\ \operatorname{dest}(k) \neq \operatorname{dest}(t) \\ \operatorname{dest}(t)=s}} \sum_{t} x_{t}^{k} \leq N_{s} \quad \forall s \in S$ 


$$
\begin{aligned}
& \sum_{k} x_{t}^{k} \geq \sum_{r} L_{t}^{r} y_{t}^{r} \quad \forall t \in T, \\
& \sum_{k} G_{k} x_{t}^{k} \leq \sum_{r} P_{t}^{r} y_{t}^{r} \quad \forall t \in T, \\
& \sum_{\substack{k \\
\operatorname{dest}(k) \neq \operatorname{dest}(t) \\
\operatorname{dest}(t)=s}} \sum_{t} G_{k} x_{t}^{k} \leq N_{s} Q_{s} \quad \forall s \in S, \\
& \sum_{\substack{r \\
i \in r}} y_{t}^{r} \leq A_{i} \quad \forall i \in I \\
& w_{k} \leq a . d_{k} \quad \forall k \in K \\
& x_{t}^{k}, y_{t}^{r}, w_{k} \in \text { Integer. }
\end{aligned}
$$

\section{Multi-objective decision-making approaches}

It is well known that there are three well-known main methods for solving a multi-objective optimization problem, namely (1) a priori articulation of preferences, in which before running the optimization algorithm, the user is able to determine the relative importance of the objective functions or desired goals, (2) a posteriori articulation of preferences, in which a single solution is selected out of the various possible solutions that are equivalent from a mathematical point of view, and (3) no articulation of preferences where the decision-maker is not able to determine any preference.

Following our discussion with the managers of the Iranian railway network, it was found that although the managers understood the importance of considering multiple objectives in the decision-making, they were not able to prioritize the objectives. This is the first time that they have introduced such an approach to the network. Consistent with the literature discussed above, we consider goal programming and $L_{p}$ norm approaches to solve our problem, in which all the weights are set to one. This is the reason why these approaches are basic methods that fall into the category of "a priori articulation of preferences" and the category of "no articulation of preferences". It is indeed a simplified version of the a priori category, in which all the weights are considered equal to one (see e.g. [23]).

In this section and for aggregation purposes, two common aggregating approaches, namely goal programming and $L_{p}$ norm, are adopted to convert a mathematical model with multiple objectives to a model with one objective.

\subsection{Goal programming approach}

In the goal programming aggregation method (see, e.g. [22]), the objective function is defined by:

$$
\min Z=\theta_{1} v_{1}^{+}+\theta_{2} v_{2}^{+}+\theta_{3} v_{3}^{-}+\theta_{4}\left(v_{4}^{+}+v_{4}^{-}\right) \text {. }
$$

In addition, the constraints below are introduced to the above developed model:

$$
\begin{aligned}
& \sum_{k} b_{k}\left(d_{k}-w_{k}\right)-\sum_{k} \sum_{t} c_{t}^{k} x_{t}^{k}-\sum_{t} \sum_{r} f_{t}^{r} y_{t}^{r} \\
& -\sum_{k} c_{k} w_{k}-\left(v_{1}^{+}-v_{1}^{-}\right)=M_{1}, \\
& \sum_{t} \sum_{r} y_{t}^{r}-\left(v_{2}^{+}-v_{2}^{-}\right)=M_{2} \\
& \sum_{s} \sum_{\substack{k \\
\operatorname{dest}(k) \neq \operatorname{dest}(t) \\
\operatorname{dest}(t)=s}} \sum_{t} x_{t}^{k}-\left(\nu_{3}^{+}-\nu_{3}^{-}\right)=M_{3}, \\
& \sum_{t} \sum_{r} P_{t}^{r} y_{t}^{r}-\sum_{k} \sum_{t} G_{k} x_{t}^{k}-\left(v_{4}^{+}-v_{4}^{-}\right)=M_{4}, \\
& v_{i}^{+}, v_{i}^{-} \geq 0 \quad \forall i \in Z
\end{aligned}
$$

where $\theta_{i}$ denotes the coefficient, $v_{i}^{+}$denotes the slack variables, and $v_{i}$ denotes the surplus variables of objective function $i . \quad M_{i}$ symbolizes the desired level (target value) for the Individual Objective Function $i$ (i.e., IOF $(i)$ ). Herein, this parameter may be assumed as the optimal Objective Function Value (OFV); the same is done here.

With regard to the following issues, the sum of the deviations of individual objective functions from the related desired level is minimized in objective function (15):

- Orientation aimed individually by each objective function (e.g., max and min);

- Individual importance factor of each objective function.

Inequalities (16) to (19) are associated with objective functions (1) to (4) by regarding their related individual level, which is desired. Constraint (20) shows that the variables of surplus and slack $\left(v_{i}^{+}\right.$and $\left.v_{i}^{-}\right)$are positive.

\section{2. $L_{p}$ norm method}

Concerning the $L_{p}$ norm technique, the aggregated mathematical goal is presented as follows:

$$
\begin{aligned}
\min L-P= & {\left[\Delta_{1}\left(\frac{Z_{1}^{*}-Z_{1}}{Z_{1}^{*}}\right)^{p}\right.} \\
& +\Delta_{2}\left(\frac{Z_{2}^{*}-Z_{2}}{Z_{2}^{*}}\right)^{p}+\Delta_{3}\left(\frac{Z_{3}-Z_{3}^{*}}{Z_{3}^{*}}\right)^{p} \\
& \left.+\Delta_{4}\left(\frac{Z_{4}-Z_{4}^{*}}{Z_{4}^{*}}\right)^{p}\right]^{\frac{1}{p}}
\end{aligned}
$$

where $\Delta_{i}$ symbolizes the multiplier of mathematical 
goal $i, Z_{i}$ symbolizes mathematical goal $i$, and $Z_{i}^{*}$ stands for the optimal OFV $i$. Objective function (21) tries to minimize the sum of normalized deviation of individual objective functions from a related solution, which is optimal, considering the following issues:

- Kind of the deviation calculation $(P)$;

- Fraction of deviation by taking into account the related individual OFV, which is optimal.

Here, objective function (21) with $p=1$ (i.e., linear) and $p=2$ (i.e., nonlinear) is considered. The set of constraints is identical with no difference.

\section{Results and sensitivity analysis}

\subsection{Experimentation of our model}

Here, ten random problems are considered. The computer utilized is characterized by Intel Core 2 Duo $2.53 \mathrm{GHz}$ CPU and 4.00 GB of RAM. The standard solver CPLEX version 12.4 is used. The incoming value $b_{k}$ is two times the value of demand loss cost $c_{k}$. The multipliers of all objectives are identical in the case of all techniques. When weights of the objectives are not pre-specified, considering identical importance values for them is not a strange task in the literature [24]. Thus, importance values (weights) that are equal to 1 are allocated to all the goals before being aware of the importance values for the objective functions. The test problems are defined in Table 1.

At first, the test problems are dealt with by taking into account each objective function individually. Table 2 demonstrates the outcomes of our numerical experiments. Here, GP and $L_{p}$ norm techniques solve these problems. Table 2 shows the values of the IOFs that are optimal. As shown in this table, the data relating to column IOF (3) are all zero. In other words, in all of Test Problems 1 to 10 (Table 1 ) the total number of shunting operations is zero, which indicates that there are no unnecessary disassembly and assembly operations (shunting operation) in the network in the

Table 1. Test problems.

\begin{tabular}{ccccccc}
\hline No. & $\begin{array}{c}\text { No. of } \\
\text { yards }\end{array}$ & $\begin{array}{c}\text { No. of } \\
\text { demands }\end{array}$ & $\begin{array}{c}\text { No. of } \\
\text { potential trains }\end{array}$ & $\begin{array}{c}\text { No. of } \\
\text { constraints }\end{array}$ & $\begin{array}{c}\text { No. of } \\
\text { variables }\end{array}$ & $\begin{array}{c}\text { Ratio of trains } \\
\text { to demands }\end{array}$ \\
\hline 1 & 30 & 25 & 427 & 2251 & 21375 & 17 \\
2 & 35 & 30 & 466 & 2688 & 27990 & 16 \\
3 & 40 & 25 & 583 & 2999 & 29175 & 23 \\
4 & 35 & 60 & 466 & 3763 & 55980 & 8 \\
5 & 50 & 45 & 991 & 5478 & 89235 & 22 \\
6 & 50 & 60 & 1257 & 7041 & 150900 & 21 \\
7 & 60 & 70 & 1380 & 8662 & 193270 & 20 \\
8 & 60 & 80 & 1475 & 9557 & 236080 & 18 \\
9 & 70 & 85 & 1475 & 10754 & 250835 & 17 \\
10 & 70 & 75 & 1380 & 9759 & 207075 & 18 \\
\hline
\end{tabular}

Table 2. Outcomes of each individual objective function, IOF, to be considered as input for GP and $L_{p}$ norm approaches employed as optimal solution (i.e., $Z_{i}^{*}$ ) or desired level (i.e., $M_{i}$ ).

\begin{tabular}{ccccc}
\hline $\begin{array}{c}\text { Problem } \\
\text { no. }\end{array}$ & $\begin{array}{c}\text { IOF (1) } \\
(\$)\end{array}$ & $\begin{array}{c}\text { IOF (2) } \\
\text { (no. of trains) }\end{array}$ & $\begin{array}{c}\text { IOF (3) } \\
\text { (no. of shunting } \\
\text { operations) }\end{array}$ & $\begin{array}{c}\text { IOF (4) } \\
\text { (tonnage) }\end{array}$ \\
\hline 1 & 368639.1 & 649.0 & 0.0 & 227533.0 \\
2 & 346586.1 & 800.0 & 0.0 & 286855.0 \\
3 & 179625.9 & 896.0 & 0.0 & 247304.0 \\
4 & 756824.6 & 839.0 & 0.0 & 533449.0 \\
5 & 411148.9 & 570.0 & 0.0 & 885869.0 \\
6 & 70344.8 & 591.0 & 0.0 & 1224201.0 \\
7 & 479483.6 & 573.0 & 0.0 & 1373561.0 \\
8 & 1409402.9 & 1112.0 & 0.0 & 1583427.0 \\
9 & 1437728.3 & 1126.0 & 0.0 & 1653359.0 \\
10 & 776791.4 & 1210.0 & 0.0 & 1503769.0 \\
\hline
\end{tabular}


optimal solution for two reasons. First, there are a high number of trains to transport demands of the problem with common origins and destination. Second, there exist demands with origin and destination different from the origin and destinations of available trains, which are rejected from transportation by the lost sale constraint (i.e., Constraint (13)). Finally, the results of the IOF (3) appear to be an ideal solution with respect to the data of this problem.

Table 3 shows the outcome of our numerical experiment. The standard solver software does not find any solution in 10 hours. The findings indicate the suitability of CPLEX in terms of time resource for GP and when $p=1$. Therefore, they are almost similar since they demonstrate the same performance. However, based on the solving time reported in Table 3 , the $L_{1}$ norm method always outperforms the goal programming method as reported in all the cases of Table 3, and the solving time related to the $L_{1}$ norm method is shorter than that related to the goal programming method (see Figure 1). However, due to

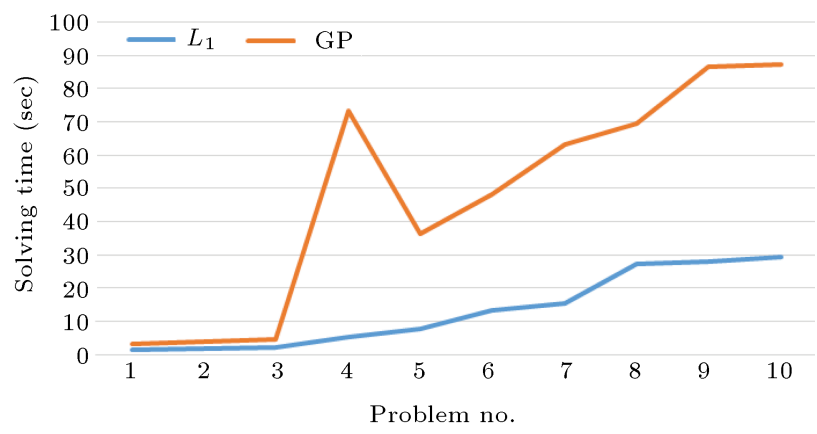

Figure 1. Comparison of the performance of GP and $L_{1}$ norm techniques, in which the $L_{1}$ norm method always outperforms the goal programming method. the non-linearity for the case $p=2$, even a feasible solution cannot be found in 10 hours for problems with a considerable size. It is worth noting that the deviation of individual objective functions in goal programming methods is not normalized; therefore, the optimal value of this method is different from that of $L_{p}$ norm method.

There are two cases for the $L_{p}$ norm technique: $p=1$ and $p=2$. Because of the non-linearity of the case $p=2$, the technique for $p=2$ is able to effectively discover better solutions in the related solution space [25]. Because of the compatibility of non-linear models with real-life conditions and their capability to attain suitable quality in solution finding, the $L_{2}$ norm method is applied. In the $L_{p}$ norm method with $p=2$, the solver has to examine more points in solution space, which calls for much more time for the discovery of a non-dominated solution. The outcomes shown in Table 3 demonstrate this argument. Regarding this issue, Figure 2(a) and (b) demonstrate the $L_{p}$ norm method in the discovery of a solution for $p=1$ and $p=2$, individually. In addition, $L_{p}$ norm with $p=1$ was merely used for testing the model and sensitivity analysis.

\subsection{Sensitivity investigation}

In order to investigate the sensitivity of our model, problem no. 3 is used in this section. This model contains multiple objective functions with different characteristics. Since each function has a different impact on the solutions, their multipliers and the target value of each individual are examined in our sensitivity analysis [26,27]. Four different aspects described below are very important, because some of the aspects are basic factors in converting the model with multiple objectives into a single combined objective one.

Table 3. Outcomes of $L_{p}$ norm and GP techniques.

\begin{tabular}{|c|c|c|c|c|c|c|}
\hline \multirow[b]{2}{*}{ No. } & \multicolumn{2}{|c|}{$L_{1}$ norm } & \multicolumn{2}{|c|}{$L_{2}$ norm } & \multicolumn{2}{|c|}{ Goal programming } \\
\hline & $\begin{array}{c}\text { Optimal } \\
\text { value* }^{*}\end{array}$ & $\begin{array}{c}\text { Solving } \\
\text { time (sec) }\end{array}$ & $\begin{array}{c}\text { Optimal } \\
\text { value }\end{array}$ & $\begin{array}{c}\text { Solving } \\
\text { time (sec) }\end{array}$ & $\begin{array}{c}\text { Optimal } \\
\text { value }\end{array}$ & $\begin{array}{c}\text { Solving } \\
\text { time (sec) }\end{array}$ \\
\hline 1 & 1.80 & 1.61 & 1.17 & 81.43 & 13954.00 & 3.24 \\
\hline 2 & 1.74 & 1.96 & 1.12 & 124.31 & 14694.00 & 3.76 \\
\hline 3 & 1.75 & 2.21 & 1.13 & 267.87 & 3883.00 & 4.68 \\
\hline 4 & 1.50 & 5.45 & $\mathrm{~N} / \mathrm{A}^{* *}$ & 36000.00 & 10304.00 & 73.26 \\
\hline 5 & 1.71 & 7.66 & 1.13 & 572.80 & 7320.00 & 36.51 \\
\hline 6 & 1.86 & 13.24 & $\mathrm{~N} / \mathrm{A}$ & 36000.00 & 10150.00 & 48.12 \\
\hline 7 & 1.53 & 15.40 & 1.09 & 36000.00 & 21648.00 & 63.31 \\
\hline 8 & 1.67 & 27.19 & $\mathrm{~N} / \mathrm{A}$ & 36000.00 & 2589.00 & 69.38 \\
\hline 9 & 1.83 & 28.11 & $\mathrm{~N} / \mathrm{A}$ & 36000.00 & 2929.00 & 86.61 \\
\hline 10 & 1.50 & 29.25 & $\mathrm{~N} / \mathrm{A}$ & 36000.00 & 11948.00 & 87.29 \\
\hline
\end{tabular}

* Optimal value $=$ The sum of deviation of individual objective functions from the related desired level $($ IOF $(i))$;

** $\mathrm{N} / \mathrm{A}=$ Not Available (i.e., no feasible solution is found in 10 hours of time). 

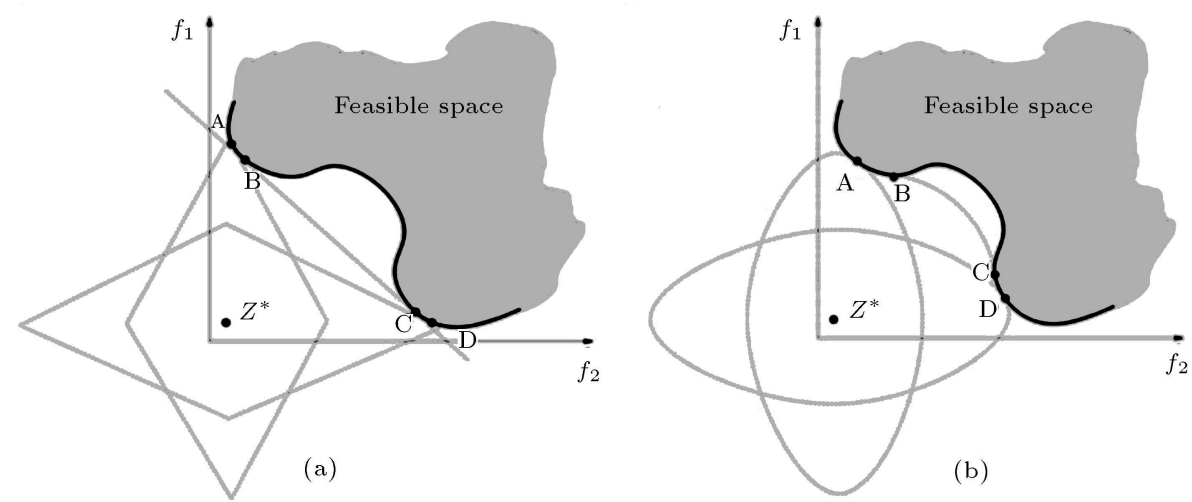

Figure 2. $L_{p}$ norm method in discovering a solution for (a) $p=1$ and (b) $p=2$.

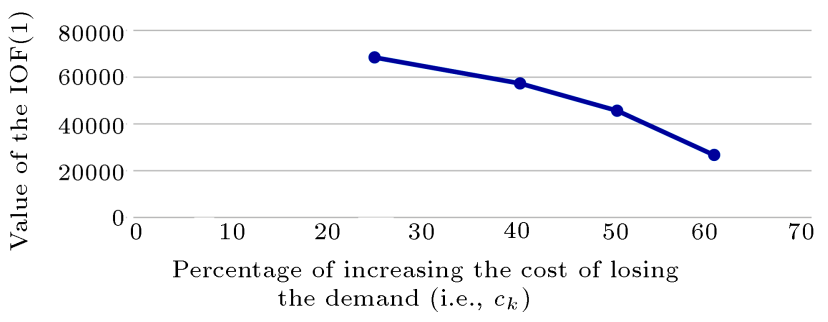

Figure 3. Impact of increasing the cost of losing the demand (i.e., $c_{k}$ ) on the value of the IOF (1).

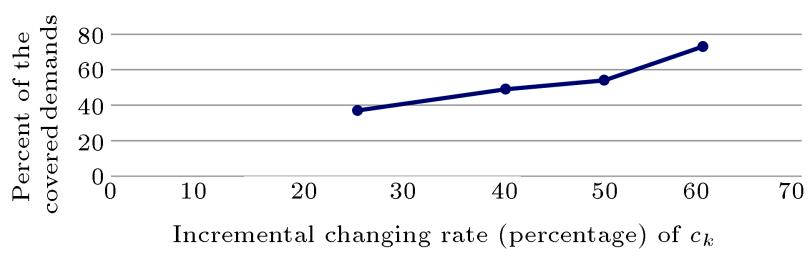

Figure 4. Impact of the incremental changing rate of the cost of losing the demand (i.e., $c_{k}$ ) on the quantity of covered demands.

\subsubsection{Impact of cost of losing demands on IOF (1)}

Figure 3 demonstrates that increasing the cost of lost demand $\left(c_{k}\right)$ leads to a significant reduction in the value of the IOF (1). As shown in Figure 2, increasing the cost of the lost demand is caused by decreasing the IOF (1), indicating a great impact on the objective function by this parameter. Figure 4 demonstrates that increasing the cost of the lost demands leads to greater demand coverage.

\subsubsection{Impact of varying the multiplier of IOFs in the $L_{1}$ norm method}

The importance factors of all IOFs are fixed as 1 . The output of the mathematical objective for the $L_{1}$ norm technique is examined by varying the importance factor of each IOF and assuming the rest are unchanged. Additionally, the importance factor of the selected IOF is fixed as 100 . Figure 5 exhibits the impact of changing the importance factor of IOFs on the mathematical

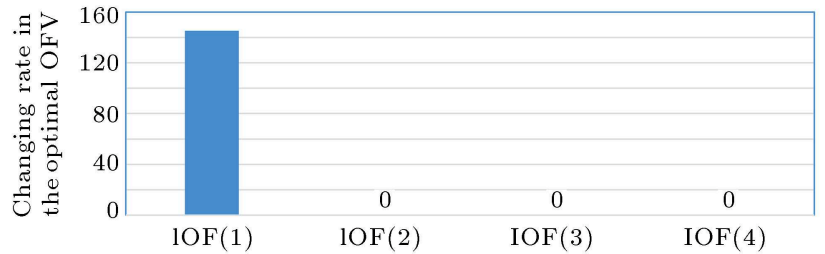

Figure 5. Impact of varying the multiplier of IOFs on the mathematical objective in the $L_{1}$ norm method.

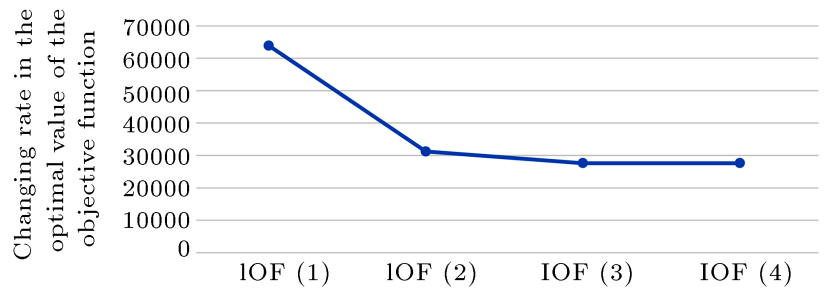

Figure 6. Impact of varying the multiplier of IOFs on the mathematical objective in a GP technique.

objective for the $L_{1}$ norm method. The outcomes demonstrate the great importance of the IOF (1).

\subsubsection{Impact of varying the multiplier of IOFs in the GP method}

To solve the problems by the GP method, the importance factors of all IOFs are fixed as 1 . The output of the mathematical objective is examined by varying the importance factor of each IOF and considering the rest as unchanged. The importance factor of the chosen IOF is fixed as 5 . Figure 6 shows the effect of varying the importance factor of IOFs on the mathematical objective in the GP method. The findings indicate significant importance of IOF (1) compared to other IOFs.

\subsubsection{Impact of changing the target value of IOFs in the GP method}

To solve the problems by the GP technique, the target values of all IOFs are set to their best values. The output of the mathematical objective is studied by 


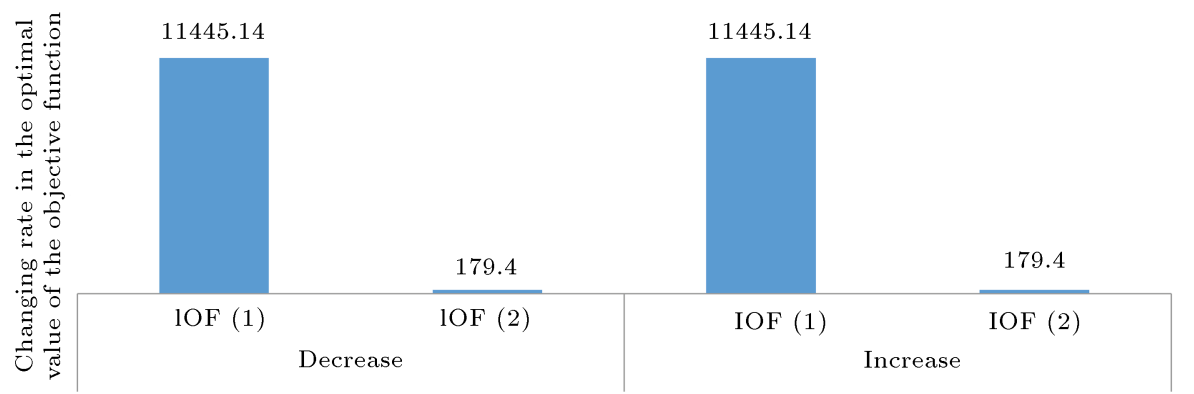

Figure 7. Impact of variations of the target value of IOFs on the best mathematical objective value in the GP technique.

varying the target value of each IOF and assuming the rest as unchanged. Figure 7 shows the impact of varying the target value of IOFs (i.e., $\pm 20 \%$ ) on the mathematical goal in the GP method. The related results illustrate the more important role of IOF (1) over other IOFs in contributing to the best output of the mathematical goal.

\section{Proposed hybrid algorithm design}

A meta-heuristic approach is adopted here to solve the model in case $p=2$ for the $L_{p}$ norm technique. The rationale behind our effort is the nonlinearity of the model in this case, which leads to unacceptable requirement for time resource for computational purposes. Specifically, a hybrid algorithm considering the SA and branch-and-cut algorithms is designed.

SA as a well-known meta-heuristics was first introduced by Kirkpatrick et al. [28]. The main steps of SA are as follows. Starting with an initial solution, SA moves to other alternative neighboring solutions iteratively. If the alternative solution outperforms the current solution, it is chosen as a new solution. If, however, the performance of the alternative solution is worse than the current solution, it is still accepted by the probability $\exp (-\Delta E / T)$, where $\Delta E$ denotes the difference of the OFV between the current and alternative neighboring solutions, and $T$ is a parameter called temperature to control the exploration process of the SA algorithm. Many iterations are executed at each temperature. Then, gradually, the temperature decreases. While, initially, the temperature is selected to be at its highest value to increase the probability of accepting undesirable solutions in terms of the OFV, the temperature has its lowest value at the end of search to decrease the probability of accepting undesirable solutions. Subsequently, the structure of our hybrid algorithm and its building blocks are explained.

In our meta-heuristic method, SA specifies the lost demand, $w_{k}$, and the exact method tackles other unknowns of the model. In Figure 7, a pseudo code for our meta-heuristic algorithm used in the problems with $p=2$ of the presented model is shown. In this pseudo code, current solution denotes the current solution, initial solution denotes the initial solution, neighbor solution denotes the neighborhood solution, and best solution denotes the best solution. In addition, Cycle denotes the number of moves needed to generate neighboring solutions. When this number reaches the defined value ( $\left.M A X_{-} C Y C L E\right)$, the internal loop of the algorithm terminates to start a new iteration with a new temperature. Afterward, the building blocks of our pseudo code are explained.

\subsection{Generating an initial solution}

To generate an initial solution, the $L_{1}$ norm technique is applied in the standard solver. In so doing, we temporarily assume a full coverage of all demands, and all of $w_{k}$ variables are set to zero. We then plug the solution from the $L_{1}$ norm technique into $L_{2}$ norm and consider its value as initial solution (see Figure 8). Since the $L_{1}$ norm technique is much more efficient than the $L_{2}$ norm technique, we apply it to find initial feasible solutions in a fast manner.

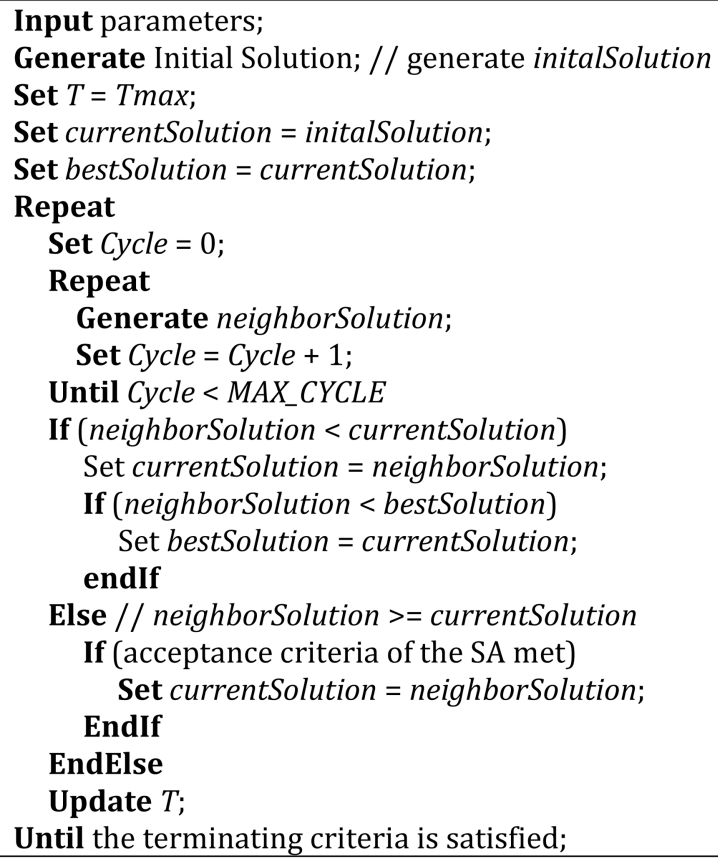

Figure 8. Pseudo code of our hybrid algorithm. 


\subsection{Generating the neighbor solutions}

The initial solution generation step leads to another step, in which a move is required to search the solution space. Figure 9 shows the pseudo code of the procedure for generating a neighbor solution. In each cycle of move, this process should be done. In this pseudo code, $M A X_{-} T E M P$ is a parameter that shows the initial temperature in $\mathrm{SA}$.

\subsection{Terminating criteria}

In the proposed algorithm, two terminating criteria are considered, namely reaching the initial temperature and $m$ numbers of reducing the temperature without any improvement.

\section{Experimental results}

For experimental purposes, our algorithm is tested on ten test cases. These test cases are already introduced in Table 1. The comparison of the behavior of the standard solver and the proposed algorithm is tabulated in Table 4. In fact, in comparison to the standard solver, our algorithm is able to provide a better solution method in terms of both problem size and speed.

Figure 10 depicts our sample investigation of a star-type railway network in Iran. This network has 60 yards. In addition, 200 origin-destination pairs demonstrate its demand pattern. Tehran plays the role of a hub in the center of the network. The yards of the network order the trains and classify them for incoming and outgoing trains, respectively.

The parameters of our sample investigation are tabulated in Table 5. Tables 6 to 8 present the results, which are similar to those of 10 test cases presented before (see Table 4). Once again, in comparison to the standard solver, our algorithm is able to provide a better solution method in terms of both problem size and speed.

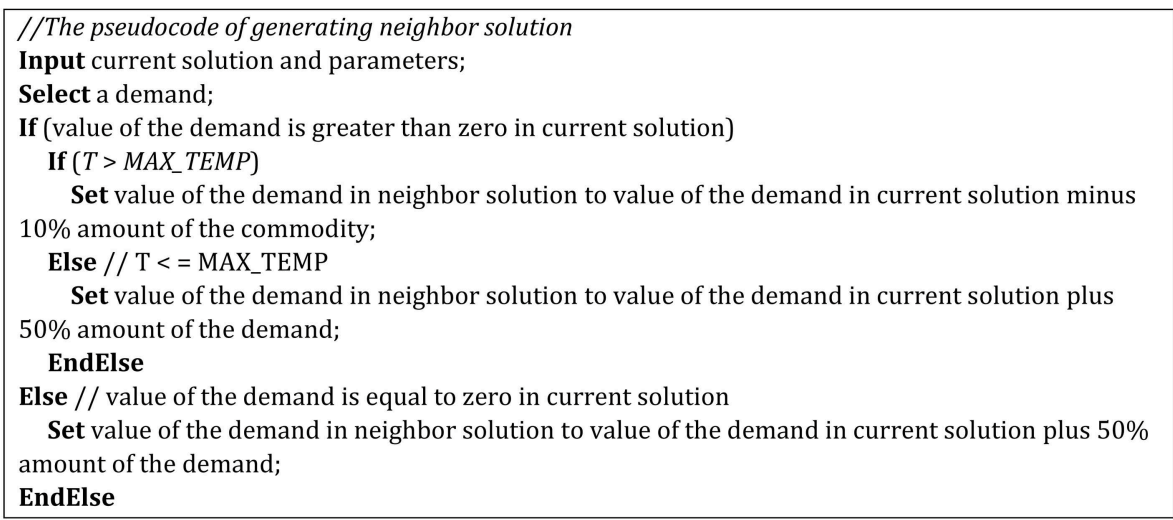

Figure 9. Pseudo code of generating the neighbor solutions.

Table 4. $L_{2}$ norm method results (i.e., $p=2$ ) by using the proposed algorithm obtained by solving test problems.

\begin{tabular}{|c|c|c|c|c|}
\hline \multirow{2}{*}{ no. } & \multicolumn{2}{|c|}{ CPLEX software } & \multicolumn{2}{|c|}{ Proposed algorithm } \\
\hline & Optimal value* & Solving time (sec) & Optimal value & Solving time (sec) \\
\hline 1 & 1.17 & 81.43 & 1.17 & 1567.87 \\
\hline 2 & 1.12 & 124.31 & 1.13 & 1391.12 \\
\hline 3 & 1.13 & 267.87 & 1.13 & 1417.25 \\
\hline 4 & $\mathrm{~N} / \mathrm{A}^{* *}$ & 36000.00 & 1.06 & 2999.83 \\
\hline 5 & 1.13 & 572.80 & 1.13 & 3190.26 \\
\hline 6 & $\mathrm{~N} / \mathrm{A}$ & 36000.00 & 1.15 & 3598.97 \\
\hline 7 & 1.09 & 36000.00 & 1.09 & 3419.57 \\
\hline 8 & $\mathrm{~N} / \mathrm{A}$ & 36000.00 & 1.10 & 3282.55 \\
\hline 9 & $\mathrm{~N} / \mathrm{A}$ & 36000.00 & 1.12 & 4055.16 \\
\hline 10 & $\mathrm{~N} / \mathrm{A}$ & 36000.00 & 1.07 & 3934.80 \\
\hline
\end{tabular}

* Optimal value $=$ The sum of deviation of individual OFVs from the related desired level (IOF (i));

** $\mathrm{N} / \mathrm{A}=$ Not Available (i.e., no feasible solution is found in 10 hours of time). 


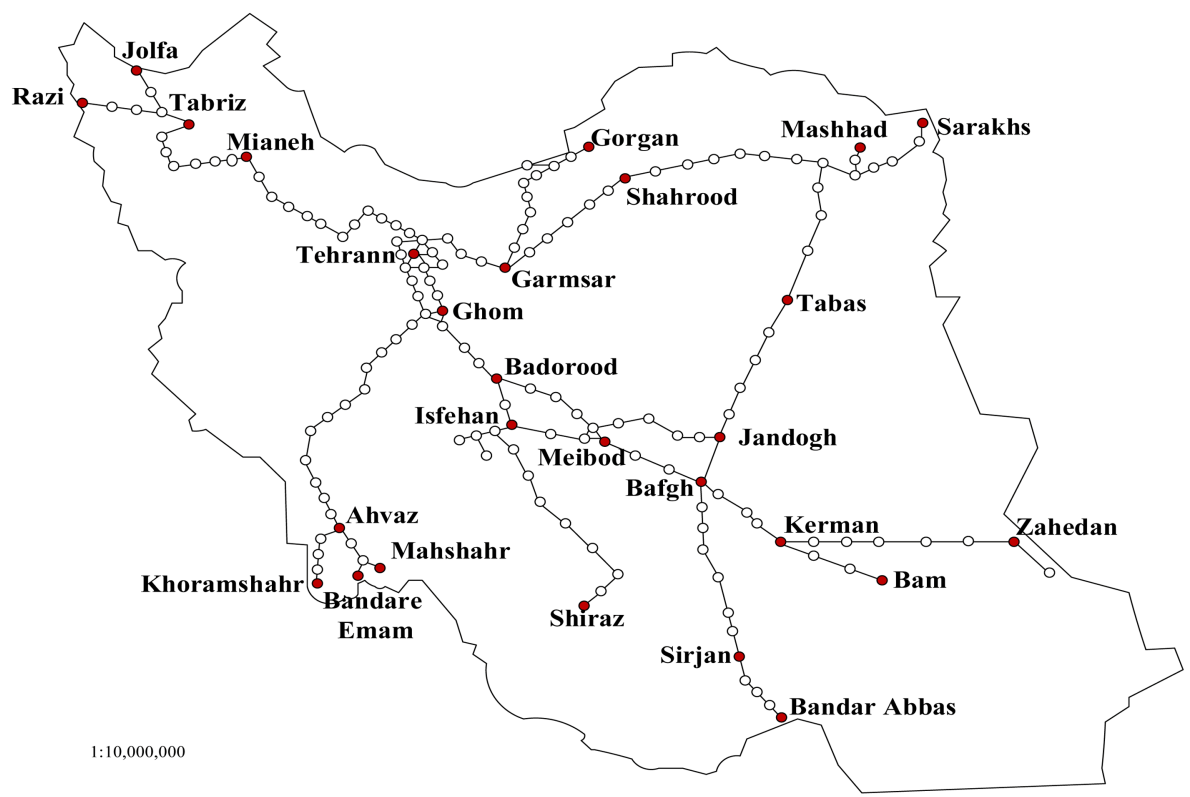

Figure 10. Network of the Iranian railway.

Table 5. Specifications of the Iranian railway.

\begin{tabular}{cccccc}
\hline $\begin{array}{c}\text { No. of } \\
\text { yards }\end{array}$ & $\begin{array}{c}\text { No. of } \\
\text { demands }\end{array}$ & $\begin{array}{c}\text { No. of } \\
\text { potential trains }\end{array}$ & $\begin{array}{c}\text { No. of } \\
\text { constraints }\end{array}$ & $\begin{array}{c}\text { No. of } \\
\text { variables }\end{array}$ & $\begin{array}{c}\text { The ratio of } \\
\text { trains to demands }\end{array}$ \\
\hline 56 & 197 & 1089 & 14534 & 429263 & 6 \\
\hline
\end{tabular}

Table 6. Outcomes of each mathematical objective to be used for GP and $L_{p}$ norm techniques employed as the optimal solution (i.e., $Z_{i}^{*}$ ) or desired level (i.e., $M_{i}$ ).

\begin{tabular}{cccc}
\hline $\begin{array}{c}\text { IOF (1) } \\
(\$)\end{array}$ & $\begin{array}{c}\text { IOF (2) } \\
\text { (number of train) }\end{array}$ & $\begin{array}{c}\text { IOF (3) } \\
\text { (number of shunting operations) }\end{array}$ & $\begin{array}{c}\text { IOF (4) } \\
\text { (tonnage) }\end{array}$ \\
\hline 281656.29 & 211.00 & 0.00 & 2529437.00 \\
\hline
\end{tabular}

Table 7. Results of the $L_{P}$ norm and GP methods for an Iranian railway network.

\begin{tabular}{cccccccc}
\hline \multicolumn{2}{c}{$\boldsymbol{L}_{\mathbf{1}}$ norm } & (i.e., $\boldsymbol{p}=\mathbf{1})$ & & \multicolumn{2}{c}{$\boldsymbol{L}_{\mathbf{2}}$ norm $($ i.e., $\boldsymbol{p}=\mathbf{2})$} & & \multicolumn{2}{c}{ Goal programming } \\
\cline { 1 - 1 } $\begin{array}{c}\text { Optimal } \\
\text { value }\end{array}$ & $\begin{array}{c}\text { Solving } \\
\text { time }(\mathbf{s e c})\end{array}$ & & $\begin{array}{c}\text { Optimal } \\
\text { value }\end{array}$ & $\begin{array}{c}\text { Solving } \\
\text { time }(\mathbf{s e c})\end{array}$ & & $\begin{array}{c}\text { Optimal } \\
\text { value }\end{array}$ & $\begin{array}{c}\text { Solving } \\
\text { time }(\mathbf{s e c})\end{array}$ \\
\hline 1.80 & 179.21 & & N/A & 36000.00 & & 496668.00 & 691.32 \\
\hline
\end{tabular}

Table 8. Computational results of $L_{2}$ norm method (i.e., $p=2$ ) by using the proposed algorithm obtained by solving an Iranian railway under study.

\begin{tabular}{ccccc}
\hline \multicolumn{2}{c}{ CPLEX software } & & \multicolumn{2}{c}{ Proposed algorithm } \\
\cline { 1 - 2 } \cline { 5 - 6 } Optimal value & Solving time (sec) & & Optimal value & Solving time (sec) \\
\hline N/A & 36000.00 & & 1.12 & 4411.29 \\
\hline
\end{tabular}

Figure 10 shows a part of the network in detail to improve the illustration. There are some demands in this part of the network. It is worth noting that the maximum number of frequencies of the train can pass through arc $i$ of network $\left(A_{i}\right)$, shown for each track of the network in Figure 11.

Table 9 defines the demands associated with
Figure 10. Here, the lower bound of the demand for each train is considered to be 10 . In addition, the upper bound of the demand for each train is assumed to be 45 .

Figure 12 shows the best plan for train formation. Here, lines show the paths passed by trains. Moreover, circles show the yards. In addition, arrows show the route of trains. The findings indicate that 17 trains 
Table 9. Demands related to Figure 10.

\begin{tabular}{|c|c|c|c|c|c|c|c|}
\hline $\begin{array}{c}\text { Demand } \\
\text { no. }\end{array}$ & Origin & Destination & $\begin{array}{c}\text { Demand } \\
\text { volume }\end{array}$ & $\begin{array}{c}\text { Demand } \\
\text { no. }\end{array}$ & Origin & Destination & $\begin{array}{c}\text { Demand } \\
\text { volume }\end{array}$ \\
\hline 1 & Chadormalo & Isfehan & 161 & 11 & Kerman & Bafgh & 18 \\
\hline 2 & Bafgh & Isfehan & 132 & 12 & Bandar Abbas & Bafgh & 32 \\
\hline 3 & Bandar Abbas & Isfehan & 76 & 13 & Qom & Isfehan & 6 \\
\hline 4 & Kerman & Isfehan & 25 & 14 & Chadormalo & Ardakan & 16 \\
\hline 5 & Tehran & Isfehan & 35 & 15 & Qom & Bafgh & 7 \\
\hline 6 & Isfehan & Sistan & 8 & 16 & Qom & Kashan & 2 \\
\hline 7 & Isfehan & Yazd & 15 & 17 & Yazd & Bafgh & 10 \\
\hline 8 & Chadormalo & Yazd & 38 & 18 & Tehran & Sistan & 14 \\
\hline 9 & Bandar Abbas & Yazd & 45 & 19 & Tehran & Kerman & 16 \\
\hline 10 & Chadormalo & Bafgh & 20 & 20 & Yazd & Isfehan & 8 \\
\hline
\end{tabular}

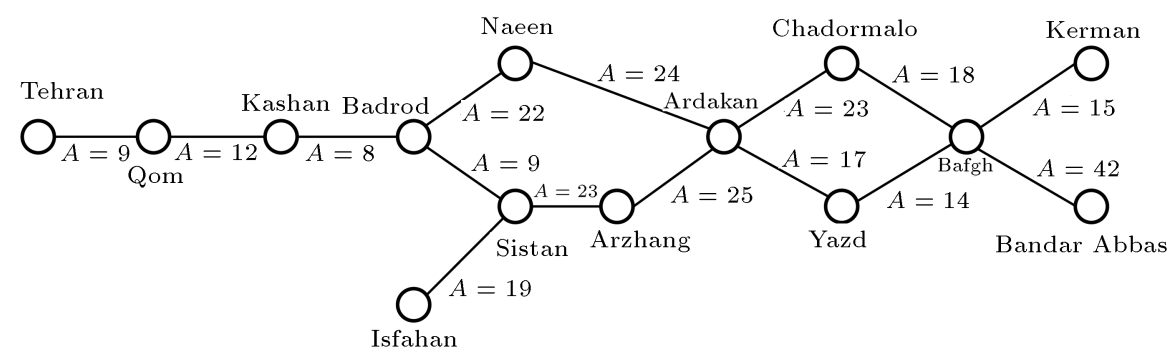

Figure 11. Part of the network to provide a more detailed illustration.

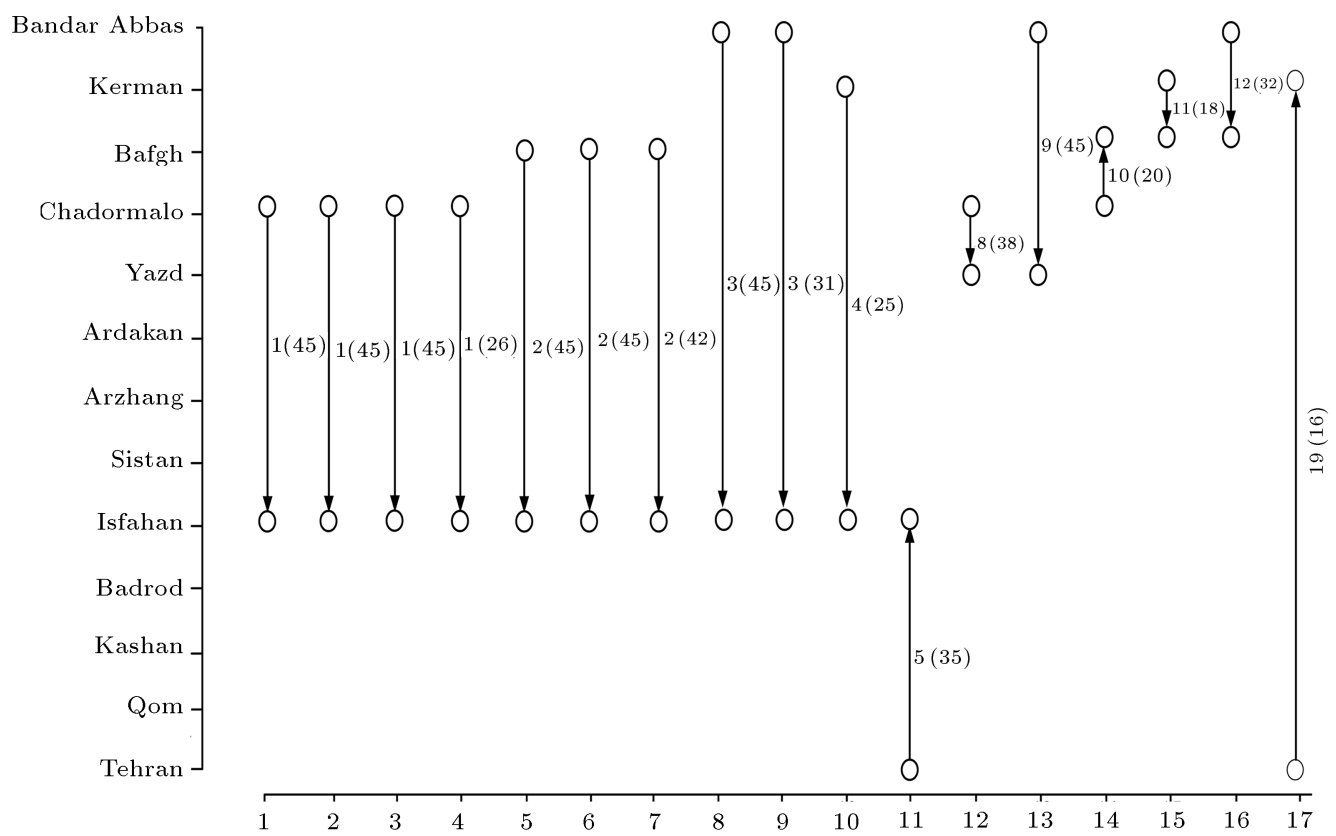

Figure 12. Train makeup and routing plan for a part of the network by the proposed algorithm.

are required to be formed. As shown in Table 9, these trains will carry 20 origin-destination pairs. Of note, as shown in Figure 11, for example, Train 8 travels through Bandar Abbas-Bafgh-Yazd-ArdakanArzhang-Sistan-Isfahan route that ships demands 3, and Train 12 travels through Chadormalo-ArdakanYazd route that carries demands 8. It is worth noting that there are some loops associated with Figure 10 in the network. For this reason, these issues cannot be shown in Figure 11. The answer shows that some of the demands are not covered (the demand of 6,7,13-18, and 20), and all of the covered demands are transferred by direct trains.

Of note, the implementation of the proposed 
model for the Iranian railway network shows that the model is completely practical and can be used for different rail networks in the world. The results reveal the capability of the proposed model.

\section{Conclusion and future work}

This study considered a mathematical model with multiple objectives for the TMP integrated routing problem. The mathematical objectives maximized the total profit, maximized customers' satisfaction level, minimized the total quantity of the operations in the yards of shunting type, and minimized the underutilized trains. To combine these objectives into only one objective, GP and $L_{P}$ norm techniques were applied. In addition, a hybrid Simulated Annealing (SA) algorithm was recommended to solve the problem. Based on the standard and proposed techniques and for experimental purposes, ten random test problems were solved. Furthermore, a sample investigation in a railway company in Iran was performed. Our algorithm was able to show nice behavior in all test cases and the sample investigation with a reasonable requirement for time resource, while the standard software could not find feasible solutions for some test cases and the sample investigation in 10 hours of time. Our findings provided significant evidence for the effectiveness of our model and proved the efficiency of our solution technique. This paper considered the customer's satisfaction as one of the key issues that will be discussed in more depth and provided more accurate parameters to measure it as the future study. Furthermore, the presented model can be extended to a routing problem by considering the time travel of trains, scheduling, and certain restrictions (e.g., safety, cost and benefit).

\section{References}

1. Berechman, J. "Urban and regional economic impacts of transportation investment: a critical assessment and proposed methodology", Transportation Research Part A: Policy and Practice, 28(4), pp. 351-362 (1994).

2. Huddleston, J.R. and Pangotra, p.p. "Regional and local economic impacts of transportation investments", Transportation Quarterly, 44(4), pp. 579-594 (1990).

3. Yaghini, M., Momeni, M., and Sarmadi, M. "A hybrid solution method for fuzzy train formation planning", Applied Soft Computing, 31, pp. 257-265 (2015).

4. Yaghini, M., Momeni, M., and Sarmadi, M. "Solving train formation problem using simulated annealing algorithm in a simplex framework", Journal of Advanced Transportation, 48(5), pp. 402-416 (2014).

5. Assad, A.A. "Modelling of rail networks: Toward a routing/makeup model", Transportation Research Part B: Methodological, 14(1), pp. 101-114 (1980).
6. Crainic, T., Ferland, J.A., and Rousseau, J.M. "A tactical planning model for rail freight transportation", Transportation Science, 18(2), pp. 165-184 (1984).

7. Keaton, M.H. "Designing railroad operating plans: A dual adjustment method for implementing Lagrangian relaxation", Transportation Science, 26(4), pp. 263279 (1992).

8. Morlok, E.K. and Thomas, E.N. Final Report on the Development of a Geographic Transportation Network Generation and Evaluation Model, Transportation Center Northwestern University (1970)

9. Huntley, C.L., Brown, D.E., Sappington, D.E., and Markowicz, B.P. "Freight routing and scheduling at CSX transportation", Interfaces, 25(3), pp. 58-71 (1995).

10. Bagheri, M., Saccomanno, F., and Fu, L. "Modeling hazardous materials risks for different train make-up plans", Transportation Research Part E: Logistics and Transportation Review, 48(5), pp. 907-918 (2012).

11. Shafia, M.A., Sadjadi, S.J., and Jamili, A. "Robust train formation planning", Proceedings of the Institution of Mechanical Engineers, Part F: Journal of Rail and Rapid Transit, 224(2), pp. 75-90 (2010).

12. Sun, Y., Cao, C., and Wu, C. "Multi-objective optimization of train routing problem combined with train scheduling on a high-speed railway network", Transportation Research Part C: Emerging Technologies, 44, pp. 1-20 (2014).

13. Masek, J., Camaj, J., and Nedeliakova, E. "Innovative methods of improving train formation in freight transport", World Academy of Science, Engineering and Technology, International Journal of Mechanical, Aerospace, Industrial, Mechatronic and Manufacturing Engineering, 9(11), pp. 1947-1950 (2015).

14. Borndörfer, R., Klug, T., Schlechte, T., Fügenschuh, A., Schang, T., and Schülldorf, H. "The freight train routing problem for congested railway networks with mixed traffic", Transportation Science, 50(2), pp. 408423 (2016).

15. Boysen, N., Emde, S., and Fliedner, M. "The basic train makeup problem in shunting yards", OR Spectrum, 38(1), pp. 207-233 (2016).

16. Cheng, J., Verma, M., and Verter, V. "Impact of train makeup on hazmat risk in a transport corridor", Journal of Transportation Safety \& Security, 9(2), pp. 167-194 (2017).

17. Bahrami, F., Safari, H., Tavakkoli-Moghaddam, R., and Modarres Yazdi, M. "On modeling door-to-door parcel delivery services in Iran", Iranian Journal of Management Studies, 9(4), pp. 883-906 (2017). 
18. Gallardo-Bobadilla, R. and Doucette, J. "A linear programming model for optimization of the railway blocking problem", Proceeding of the American Railway Engineering and Maintenance-of-Way Association Annual Conference (AREMA 2014), Chicago, IL, September 28 - October 1 (2014).

19. Yaghini, M., Momeni, M., and Sarmadi, M. "An improved local branching approach for train formation planning", Applied Mathematical Modelling, 37(4), pp. 2300-2307 (2013).

20. Jamili, A. "A mathematical model and a hybrid algorithm for robust periodic single-track train-scheduling problem", International Journal of Civil Engineering, 15(1), pp. 63-75 (2017).

21. Tavakkoli-Moghaddam, R., Safaei, N., and Sassani, F. "A new solution for a dynamic cell formation problem with alternative routing and machine costs using simulated annealing", Journal of the Operational Research Society, 59(4), pp. 443-454 (2008).

22. Alikhani-Kooshkak, R., Tavakkoli-Moghaddam, R., Jamili, A., and Ebrahimnejad, S. "Solving a multiobjective train makeup model with locomotive limitation by a firefly algorithm: A case study", Proc. of the Institution of Mechanical Engineers, Part F: Journal of Rail and Rapid Transit, 232(5), pp. 14831499 (2018).

23. Hwang, C.L. and Masud, A.S.M., Multiple Objective Decision Making-Methods and Applications: A stateof-the-Art Survey, (164). Springer Science \& Business Media (2012).

24. Coello Coello, C.A. and Christiansen, A.D. "MOSES: A multi-objective optimization tool for engineering design", Engineering Optimization, 31(3), pp. 337-368 (1999).

25. Evans, G.W. "An overview of techniques for solving multi-objective mathematical programs", Management Science, 30(11), pp. 1268-1282 (1984).

26. Homma, T. and Saltelli, A. "Importance measures in global sensitivity analysis of nonlinear models", Reliability Engineering \& System Safety, 52(1), pp. 117 (1996).

27. Saltelli, A., Chan, K., and Scott, E.M. (Eds.), Sensitivity Analysis, 1, New York: Wiley (2000).

28. Kirkpatrick, S., Gelatt, C.D., and Vecchi, M.P. "Optimization by simulated annealing", Science, 220(4598), pp. 671-680 (1983).

\section{Biographies}

Reza Alikhani is a PhD student at the School of Industrial Engineering at Islamic Azad University, South Tehran Branch Tehran Iran. His main research interests are in the fields of rail transportation planning, supply chain management, logistic management, and transportation management.

Reza Tavakkoli-Moghaddam is a Professor of Industrial Engineering at University of Tehran in Iran. He obtained his $\mathrm{PhD}$ in Industrial Engineering from the Swinburne University of Technology in Melbourne (1998). He is an Associate Member at Academy of Sciences in Iran and serves as Editorial Board of the International Journal of Engineering and Iranian Journal of Operations Research. He was the recipient of the 2009 and 2011 Distinguished Researcher Award and the 2010 Distinguished Applied Research Award at the University of Tehran, Iran. He was selected as a National Iranian Distinguished Researcher for 2008 and 2010 in Iran. He has published 4 books, 24 book chapters, and more than 1000 journal and conference papers.

Amin Jamili is an Assistant Professor in School of Industrial Engineering, College of Engineering, at University of Tehran in Iran. He received his PhD in Industrial Engineering from Iran University of Science and Technology. Moreover, he received his BSc and MSc degrees both from Iran University of Science and Technology and Sharif University of Technology, respectively. His research interest areas include rail transportation planning, train scheduling, and optimization under uncertainty. He has published a number of papers in journals and conferences.

Sadoullah Ebrahimnejad is an Associate Professor of Industrial Engineering at Islamic Azad University Karaj Branch, Karaj, Iran. His research interests include operations management, risk management, fuzzy MCDM models, mathematical modelling, production and supply chain management, DEA, multi-criteria networks, and meta-heuristics algorithms. 